\title{
Ethical considerations for critical care research
}

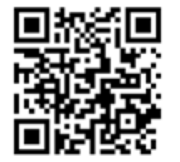

Ethical lapses are almost never a case of bad people, doing bad things, for no good reason. More often they are good people, doing bad things, for good reasons. (Marcia Angell, previous Editor-in-Chief (1988 - 2000), New England Journal of Medicine.)

Critical illness carries high morbidity and mortality worldwide, with a disproportionate burden of critical illness in low- and middle-income countries, where access to intensive care is particularly limited. ${ }^{\text {[1] With- }}$ out research in the intensive care unit (ICU) population, we are unlikely to improve our understanding of how to safely and effectively manage a wide range of diseases and injuries, minimise discomfort, reduce organ dysfunction, improve survival, improve quality of life in survivors of critical illness, and ensure rational and equitable use of scarce resources. Clinical research in the critical care environment is therefore essential to inform best practice ('evidence-based care').

The Southern African Journal of Critical Care (SAJCC) is committed to publishing clinical research in critical care, with the proviso that the research is conducted and presented in an ethically appropriate manner. Critical care research has special ethical challenges. Many critically ill patients are essentially captive and entirely dependent on the ICU team for their care. Their critical illness may mean that their ability to tolerate an adverse event from an experimental intervention, such as excessive blood sampling, could be compromised. In addition, their condition, medication and presence of invasive ventilation may make communication and/or understanding difficult, and affect a patient's ability to make rational informed decisions. Most ICU patients are therefore in no position to give informed consent. Proxy consent by a relative may also be compromised by anxiety for their relative, making them susceptible to pressure from the ICU management team. In this context, a balance is clearly needed between discovering new knowledge and protecting patients from research-related risk of harm. ${ }^{[2]}$ These aspects, among others, make ICU patients particularly vulnerable to research-related exploitation, coercion and risk of harm. ${ }^{[3]}$

It is, however, generally accepted that research can and should be conducted in vulnerable population groups, provided additional safeguards are put in place to minimise the risk of research-related harm and exploitation. These include the 'subject-condition' requirement, whereby participants must have the condition being investigated, and the 'necessity' requirement, where there must be assurance that the research could not feasibly or appropriately be conducted in a less vulnerable group. ${ }^{[4]}$

In order to ensure that the ethical integrity of critical care research is maintained, a number of ethical principles must be considered. The Belmont report ${ }^{[]]}$presents three fundamental concepts for ethical research: respect for persons, justice, and beneficence (or its corollary non-maleficence). The principle of respect for persons includes the obligation to treat the subject as an autonomous agent, for example by obtaining their informed consent for participation in research. Beneficence refers to the requirement that researchers act to maximise potential benefits to participants while minimising associated risks.

Emanuel et $a .^{[8]}$ expanded these principles in listing the requirements for ethical research. These are discussed below in the context of ICU research. ${ }^{[9]}$

\section{Societal value}

Considering the global burden of critical illness (both acutely and after discharge), most research with the objective to improve critical care within the community concerned has clear societal benefit, provided such research can be used to improve clinical outcome.

\section{Scientific rigour and validity}

Poor science equates to poor ethics, although these concepts are sometimes confused. If research is poorly conducted or methodologically unsound, it cannot be said to be generalisable. This could place participants at risk of research-related harm without any personal or societal benefit (i.e. it would not be able to inform practice), which shifts the risk-benefit ratio to unfavourable.

In order for a novel intervention to be compared with either placebo or current care, there is the ethical requirement for equipoise - this is defined as a genuine uncertainty in the expert medical community on whether an intervention is beneficial to patients. Equipoise is the ethical principle underlying randomisation to different treatment arms. ${ }^{[9]}$

\section{Acceptable risk-benefit ratio}

Similar to clinical decision-making processes, any research interventions for which the benefits equal or exceed the risks are generally considered ethically acceptable. In some cases a small net risk may be permissible in order to generate knowledge to benefit future patients. ${ }^{[2,10]}$

\section{Informed consent}

Informed consent upholds the principle of respect for autonomy, and, together with independent review, is one of the cornerstones of ethical research.

Informed consent, which is required for most prospective, interventive research, is sometimes difficult to obtain correctly in the ICU context. The informed consent process is designed as a mechanism for the participant to protect themselves. In order for consent to be valid, potential participants must: have intact decision-making capacity; be legally competent; be fully informed; be able to communicate a decision (not necessarily verbally); and offer the consent voluntarily, without any implicit or explicit coercion or undue influence. ${ }^{[11]}$

Critically ill or injured patients may not be able to understand the information provided to them or have sufficient decisionmaking ability, owing to, among others, the underlying illness, delirium or medication (e.g. sedatives, opioids). The presence of invasive ventilation does not in itself equate to limited capacity, and in ventilated patients the degree of decision-making capacity should therefore be assessed before consent is requested. When communication is limited, researchers could consider taking assent from the participant themselves (indicating some degree of understanding and agreement), as well as proxy consent, similar to what is done in most paediatric research. ${ }^{[3,6,11]}$

In critically ill patients without the capacity to provide independent consent, proxy consent may be obtained from the person's legally authorised representative (e.g. from an advance directive), a spouse or family member. The person providing consent must be properly acquainted with the participant and should have no conflicts of interest. Proxy consent is given using either the 'substituted judgement' standard, where the decision is based on a good-faith judgement of what the participant would have chosen if he/she were able to make the decision him-/herself, or a decision made in the 'best interests' of the participant. ${ }^{[6]}$ 
It is important that consent forms are written in plain language, avoiding medical jargon, and that they clearly describe the potential benefits and risks of the study. Consent forms must be provided in a language understood by the person giving consent and a translator must be on hand if the investigator taking the consent is not able to speak the consenter's language. ${ }^{[1]}$

Some categories of research may be awarded a waiver of the need for informed consent following research ethics committee review, for example where the research holds only minimally increased risks above standard care, where important research would not feasibly be possible without the waiver, and in emergency research situations. ${ }^{[12]}$ Silverman et al. ${ }^{[1]}$ provide a template for informed consent in the ICU, which is a useful resource for researchers to adapt to local context.

Research into emergency conditions, such as cardiac arrest, where prospective consent may not be possible, is particularly challenging. In some situations deferred consent, where consent is obtained retrospectively, may be permitted by the ethics review board. For example in the FIRST trial conducted in Cape Town, deferred consent was permitted because the two resuscitation fluids studied were both already in clinical use, there was equipoise, and the study outcome was considered of sufficient benefit to society to counterpoise potential subject harm. ${ }^{[13]}$

\section{Fair selection of participants}

This relates to the ethical principle of justice. Vulnerable critically ill populations should only be included in research where absolutely necessary, but they should also not be excluded from research which might be beneficial to them and/or future ICU patients.

\section{Independent review}

All research involving human participants requires independent review by a research ethics committee or institutional review board. This includes the retrospectivecollection of data from medical records, prospective observational studies and interventional trials. Articles describing human research that have not undergone ethical review will generally not be considered for publication in peer-reviewed journals, including SAJCC, and are considered unethical. Reports of ICU practice improvement initiatives that have not systematically collected individual patient data for the purposes of research may be considered exempt from ethical review. Similarly, case studies and small case series (usually $<5$ patients) may be exempt from ethical review, but consent should be obtained from the included patients for the use of their data, and this must be stated in the research paper.

\section{Respect for potential and enrolled subjects}

Participants should have the right to withdraw from a study or not to participate without affecting the medical care they receive in ICU. This must be explicitly stated in the informed consent document, and during the informed consent process.
Clinical research should be distinguishable from clinical care. Clinical care has a personalised focus directed at helping a particular person in need of expert medical attention. The purpose of clinical research, however, is to develop generalisable knowledge, which may or may not benefit the individual participant. ${ }^{[1]}$ An inability to fully understand the difference between research and clinical practice is termed therapeutic misconception. Participants may conflate research participation with receiving 'cutting-edge' care, with the unwarranted belief or hope of benefit. Therapeutic misconception is common in the critical care environment, where most critical care health providers, who are first and foremost clinicians with the intent of providing best care to their patients, frequently fulfil a dual role of care provider and researcher. ${ }^{[6]}$

\section{Conclusion}

Clinical research is essential to ensure optimal and safe ICU management. Ethical compliance is fundamental to ensuring that we effectively protect these vulnerable patients and ensure best practice through research, rather than protecting them from research.

\section{B Morrow}

Department of Paediatrics and Child Health, University of Cape Town, South Africa

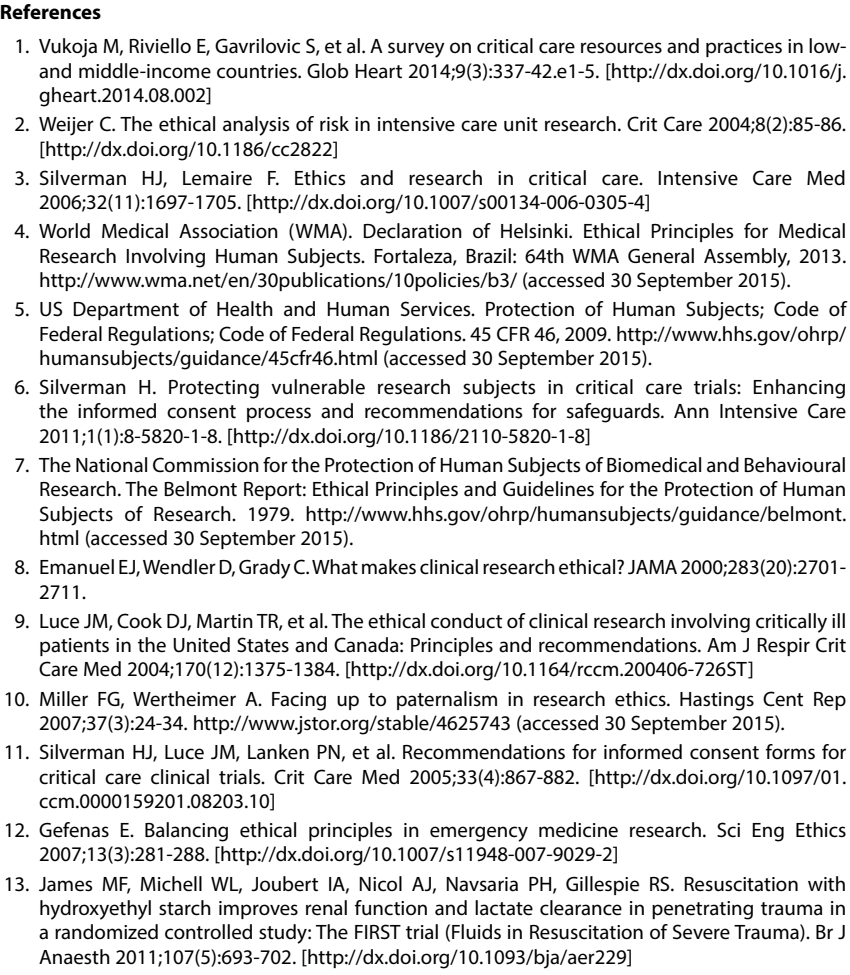

1. Vukoja M, Riviello E, Gavrilovic S, et al. A survey on critical care resources and practices in lowand middle-income countries. Glob Heart 2014;9(3):337-42.e1-5. [http://dx.doi.org/10.1016/j. gheart.2014.08.002]

2. Weijer C. The ethical analysis of risk in intensive care unit research. Crit Care 2004;8(2):85-86. [http://dx.doi.org/10.1186/cc2822]

3. Silverman HJ, Lemaire F. Ethics and research in critical care. Intensive Care Med 2006;32(11):1697-1705. [http://dx.doi.org/10.1007/s00134-006-0305-4]

4. World Medical Association (WMA). Declaration of Helsinki. Ethical Principles for Medical Research Involving Human Subjects. Fortaleza, Brazil: 64th WMA General Assembly, 2013. http://www.wma.net/en/30publications/10policies/b3/ (accessed 30 September 2015).

5. US Department of Health and Human Services. Protection of Human Subjects; Code of Federal Regulations; Code of Federal Regulations. 45 CFR 46, 2009. http://www.hhs.gov/ohrp/ humansubjects/guidance/45cfr46.html (accessed 30 September 2015).

6. Silverman H. Protecting vulnerable research subjects in critical care trials: Enhancing the informed consent process and recommendations for safeguards. Ann Intensive Care 2011;1(1):8-5820-1-8. [http://dx.doi.org/10.1186/2110-5820-1-8]

7. The National Commission for the Protection of Human Subjects of Biomedical and Behavioural Research. The Belmont Report: Ethical Principles and Guidelines for the Protection of Human Subjects of Research. 1979. http://www.hhs.gov/ohrp/humansubjects/guidance/belmont. html (accessed 30 September 2015).

8. Emanuel EJ, Wendler D, Grady C. What makes clinical research ethical? JAMA 2000;283(20):27012711.

9. Luce JM, Cook DJ, Martin TR, et al. The ethical conduct of clinical research involving critically ill patients in the United States and Canada: Principles and recommendations. Am J Respir Crit Care Med 2004;170(12):1375-1384. [http://dx.doi.org/10.1164/rccm.200406-726ST]

10. Miller FG, Wertheimer A. Facing up to paternalism in research ethics. Hastings Cent Rep 2007;37(3):24-34. http://www.jstor.org/stable/4625743 (accessed 30 September 2015).

11. Silverman $\mathrm{HJ}$, Luce JM, Lanken PN, et al. Recommendations for informed consent forms for critical care clinical trials. Crit Care Med 2005;33(4):867-882. [http://dx.doi.org/10.1097/01. ccm.0000159201.08203.10]

12. Gefenas E. Balancing ethical principles in emergency medicine research. Sci Eng Ethics 2007;13(3):281-288. [http://dx.doi.org/10.1007/s11948-007-9029-2]

13. James MF, Michell WL, Joubert IA, Nicol AJ, Navsaria PH, Gillespie RS. Resuscitation with hydroxyethyl starch improves renal function and lactate clearance in penetrating trauma in a randomized controlled study: The FIRST trial (Fluids in Resuscitation of Severe Trauma). Br Anaesth 2011;107(5):693-702. [http://dx.doi.org/10.1093/bja/aer229]

S Afr J Crit Care 2015;31(2):34-35. DOI:10.7196/SAJCC.2015.v31i2.249

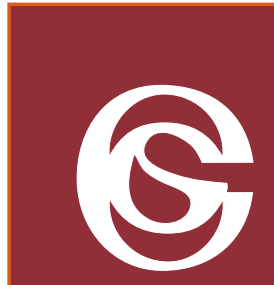

The Critical Care Society of Southern Africa works for the benefit of critically ill patients. Membership is open to all healthcare professionals involved in the management of the critically ill. Visit the Society's web page at: www.criticalcare.org.za 